\title{
Does Post-Biopsy Mammography at Short-Term Interval Contribute to Early Detection of Cancer in Patients Diagnosed with Benign-Concordant Microcalcifications on Stereotactic Biopsy?
}

\author{
Go Eun Yang ${ }^{1,2}$, Eun-Kyung Kim ${ }^{1}$, Min Jung Kim ${ }^{1}$, Hee Jung Moon ${ }^{1}$, Vivian Youngjean Park ${ }^{1}$ and Jung \\ Hyun Yoon ${ }^{1, *}$ \\ ${ }^{1}$ Department of Radiology, Severance Hospital, Research Institute of Radiological Science, College of Medicine, Yonsei University, Seoul, South Korea \\ ${ }^{2}$ Department of Radiology, Kangwon National University, Chuncheon, South Korea \\ "Corresponding author: Department of Radiology, Severance Hospital, Research Institute of Radiological Science, College of Medicine, Yonsei University, Seoul, South Korea. \\ Email: lvjenny@yuhs.ac
}

Received 2018 May 04; Revised 2019 February 27; Accepted 2019 March 02.

\begin{abstract}
Background: Currently, there are no strict post-biopsy guidelines for managing stereotactic biopsy-proven benign lesions manifesting as microcalcifications on mammography without an accompanying mass.

Objectives: To evaluate whether short-term follow-up mammography contributes to the detection of early cancer for patients with microcalcification-only lesions that were diagnosed as benign stereotactic biopsy.

Patients and Methods: Two-hundred sixty-two microcalcification-only lesions in 259 women (mean age: 47.7 years) who were diagnosed as benign on stereotactic biopsy and been followed with mammography for at least 2 years were included. Serial mammograms were reviewed from the initial pre-biopsy mammograms to the most recent examination. Mammographic findings were designated as stable, decreased, or newly developed microcalcifications. Medical records of each patient were reviewed for clinical information and pathology results of additional biopsy or surgical procedures.

Results: Of the 262 microcalcifications diagnosed as benign on stereotactic biopsy, 258(98.5\%) were finally diagnosed as benign, one (0.4\%) as high-risk lesion, and three (1.1\%) as malignant. The three cancers were all diagnosed as ductal carcinoma in situ (DCIS) at 31, 36 and 6 months after initial biopsy. The DCIS diagnosed at 6 months post-biopsy had no mammographic changes, and was detected on breast ultrasonography, while the remaining two cases had no detectable findings on the short-term follow-up mammography. Conclusion: Short-term follow-up mammography did not contribute in detecting additional breast cancers, and may have a limited role in patients diagnosed with benign-concordant microcalcifications on large-bore stereotactic biopsy.
\end{abstract}

Keywords: Breast, Biopsy, Stereotactic Biopsy, Mammography, Microcalcifications

\section{Background}

Breast biopsy under imaging guidance is nowadays widely accepted as an alternative to excisional biopsy because not only is it accurate, less invasive with minimal scarring, and cost-effective $(1,2)$, it enables avoiding second therapeutic surgeries for patients who are proven as cancer on biopsy. According to the type of lesion detected, the imaging modality that is considered to be most appropriate for guidance is used to maximize the diagnostic accuracy of the biopsy procedures. For masses that are visualized on breast ultrasonography (US), US-guided core needle biopsy is commonly considered, showing high diagnostic accuracy and sensitivity, 98.4\% and 96.3\%, respectively $(2,3)$. As for lesions manifesting as microcalcifica- tions on mammography without an accompanying mass, stereotactic biopsy under mammography guidance is usually performed, since visualization and accurate targeting of microcalcifications is difficult using US (4).

As for post-biopsy management, short-term follow-up after biopsy is usually recommended for lesions with benign results to avoid overlooking false-negative biopsy results. Imaging follow-up intervals vary among institutions, ranging from 6 to 12 months (5-9), and currently there are no strict post-biopsy guidelines for managing biopsyproven benign breast lesions. Recently, several reports suggested that short-term imaging follow-up of 6 months is unnecessary for asymptomatic benign-concordant breast lesions, since similar cancer detection rates were observed between patients with short-term follow-up and those 
with annual follow-up (6, 8-13). Yet, most of these studies evaluated the outcomes of either US-guided biopsies or stereotactic biopsies of masses, and little has been evaluated focusing on the outcome of microcalcification-only lesions diagnosed as benign on mammography-guided stereotactic biopsy.

\section{Objectives}

Based on this, the purpose of this study was to evaluate whether short-term follow-up mammography contributes to the detection of early cancer for patients with microcalcification-only lesions that were diagnosed as benign stereotactic biopsy.

\section{Patients and Methods}

This retrospective study has been approved by our institutional review board (IRB), and neither patient approval nor informed consent was required for review of images or medical records. Informed consent was obtained from all patients prior to biopsy or surgery.

\subsection{Patients}

From January 2009 to December 2014, 643 breast microcalcification-only lesions detected on mammography in 622 women had undergone mammography-guided stereotactic vacuum-assisted biopsy. Among them, 456 microcalcifications in 445 women were diagnosed as benign, which was considered concordant to the imaging features. Of these patients, 16 were excluded since they had surgery after biopsy without follow-up images, 88 were excluded due to the lack of follow-up, and 90 were excluded since they had been followed for less than 2 years after biopsy. Finally, 262 microcalcifications in 259 women were included in this study. The mean age of the 259 women were 47.7 (range, 25 to 82 ) years. Mean follow-up interval from biopsy to the most recent follow-up mammography was 55.0 (range: 23.3 to 95.9) months.

\subsection{Mammography Interpretation}

Mammograms were performed with one of two dedicated digital mammography units (Senographe DS, GE Medical Systems, Milwaukee, WI; Lorad Selenia, Hologic, Danbury, CT). Standard mediolateral oblique (MLO) and craniocaudal (CC) mammograms and magnification views with $90^{\circ}$ lateral and craniocaudal projections were obtained for all patients. During the study period, one radiologist among 20 radiologists dedicated to breast imaging (four staff radiologists, 20 fellows, range of experience: 1 to 15 years) independently reviewed the mammograms and categorized the microcalcifications. One radiologist with 8 years of experience in breast imaging (J.H.Y.) retrospectively reviewed the mammograms and analyzed the imaging features according to the 5th edition of the American College of Radiology Breast Imaging Reporting and Data System (ACR BI-RADS) (14).

\subsection{Biopsy Procedures}

Microcalcifications that were considered as suspicious on mammography were targeted for biopsy. In cases of multiple or extensive microcalcifications, the area showing most suspicious morphology or the largest number of microcalcifications were targeted.

Stereotactic biopsy was performed with the patient positioned in lateral decubitus position on the biopsy table (Mammotome ST, Ethicon Endo-Surgery Inc., Cincinnati, $\mathrm{OH}, \mathrm{USA})$. Biopsies were performed using either 11-gauge or 8-gauge vacuum-assisted biopsy probes, needle size was decided upon according to the extent of microcalcifications, breast thickness, and the preference of the performing radiologist. During the study period, three radiologists dedicated to breast imaging performed stereotactic biopsy, each with 8 - 15 years of experience. Local anesthesia was routinely applied using $1 \%$ lidocaine. Five or more specimens were routinely retrieved during biopsy, and specimen mammography was obtained to see if the targeted microcalcifications had been sufficiently extracted from the targeted area. Stereotactic biopsy of microcalcifications was considered adequate (1) if microcalcifications were detected on specimen mammography, and (2) if the presence of microcalcifications was described on the pathology reports. In cases that microcalcifications had been completely extracted or the knowledge of the biopsy site was critical in deciding upon surgical management, post-biopsy clip (MammoMARK, Devicor Medical Products) was placed immediately after biopsy procedures.

Routine mammography of the biopsied breast (MLO and CC views) were obtained approximately 1 week after biopsy at our institution to evaluate (1) post-biopsy complications such as hematomas, (2) extent of the remnant microcalcifications, (3) and the location of the post-biopsy clip, if inserted.

\subsection{Image-Histology Correlation}

On the regular weekly conferences held in our breastimaging department, mammograms of the patients that had undergone stereotactic biopsy for suspicious microcalcifications were reviewed, and decided upon concordance between the imaging features of the microcalcifications and the pathologic diagnosis. Discordant biopsy results were defined as pathology results that were insuffi- 
cient to explain the microcalcifications seen on mammography, and for these calcifications, additional diagnostic surgery was recommended. Imaging follow-up with mammography was recommended for benign-concordant microcalcifications, at 6, and 12 months after biopsy, then yearly if stable.

\subsection{Data and Statistical Analysis}

Post-biopsy mammography performed at 4 - 9 month interval was defined as short-term imaging follow-up (10). Pathologic results from biopsy were classified into definitive diagnosis, that is, specific pathologic entity that could account for the lesion, including fibroadenoma, fibroadenomatous hyperplasia, tubular adenoma, hematoma or inflammation, abscess, hamartoma, fat necrosis, granulomatous disease, lymph node, myofibroma, cyst contents, pseudoangiomatous stromal hyperplasia (PASH), duct ectasia. Benign, nonspecific results included fibrocystic changes, adenosis, columnar cell change, fibrous mastopathy, apocrine metaplasia, duct hyperplasia, and stromal fibrosis (9).

Chi-square test or Fisher's exact test was used for comparing categorical variables. Independent $t$-test was used to compare continuous variables. Statistical analysis was done using PASW version 20 (IBM Corporation, Somers, NY, USA). P values of less than 0.05 were considered to have statistical significance.

\section{Results}

Among the 262 microcalcifications in the 259 women included in our study, 258 microcalcifications (98.5\%) were finally diagnosed as benign, one $(0.4 \%)$ as high-risk lesion (ADH), and three (1.1\%) as malignant. Of the 259 women, 194 (74.9\%) microcalcifications had short-term follow-up mammography. Table 1 summarizes the demographics and pathologic findings of the 262 microcalcifications included in this study. The majority of benign-concordant microcalcifications were assessed as BI-RADS category $4 \mathrm{a}$ ( $n=236,90.1 \%$ ), prior to biopsy. There were no significant differences between the presences of short term follow-up images according to BI-RADS final assessment category $(\mathrm{P}=$ 0.471). According to the pathologic diagnosis from biopsy, 20 (7.6\%) cases had specific diagnosis and 242 (92.4\%) had nonspecific diagnosis, and the patients with short-term follow-up mammography had significantly higher rates of non-specific pathologic diagnosis, $94.8 \%$ to $85.3 \%$ ( $\mathrm{P}=$ 0.022 ).

Among the 262 biopsy-proven benign microcalcifications, four (1.5\%, 4 of 262 ) cases showed changes on follow-up mammography, either increased number of preexisting microcalcifications on mammography or newly developed lesions on breast ultrasonography (US) at the same quadrant of the prior biopsy site (Table 2). One case was diagnosed as 4-mm sized atypical ductal hyperplasia on surgery performed on the newly developed microcalcifications detected on mammography 5 months after stereotactic biopsy. One case that had been diagnosed as malignancy 6 months after biopsy had a suspicious mass detected on breast US located in the same quadrant as the microcalcifications targeted for prior stereotactic biopsy. No evident changes were seen on the follow-up mammography performed after stereotactic biopsy (Figure 1). The other two cases had newly developed microcalcifications on follow-up mammography, which was considered suspicious requiring additional biopsy procedures (Figure 2). All four cases that showed changes on follow-up breast imaging after stereotactic biopsy had nonspecific pathologic diagnosis on initial stereotactic biopsy.

\section{Discussion}

In total, the malignancy rate for the benignconcordant microcalcifications in this study was $1.1 \%$ (3/262), which is within the $<2 \%$ range recommended for ACR BI-RADS category 3 assessment (14). Our results are also similar to the results of previous studies including biopsyproven benign lesions detected on US or mammography $(6,11-13)$. In the prior studies $(6,11-13)$, the type of breast lesion or the imaging modality used for guidance was not limited to a specific type, showing low malignancy rates for biopsy-proven benign lesions in general. In particular, for cases representing as microcalcifications-only without associated palpable lesions targeting for biopsy among the microcalcifications, distribution of the lesions broadly among the breast critically affects the diagnostic accuracy of stereotactic biopsy. Based on this, we hypothesized that microcalcification-only lesions diagnosed as benign on stereotactic biopsy may have higher false-negative results. Results of our study revealed a low malignancy rate of the benign-concordant microcalcification-only lesions consistent to prior studies, supporting that these microcalcifications are at low risk for malignancy and could be considered safe for follow-up.

Usually, imaging follow-up is recommended for benign-concordant breast lesions to avoid any delayed diagnosis of a possible false-negative biopsy (15), but at present there are no standardized imaging follow-up guidelines that specify a follow-up interval or duration for lesions (8). Results of recent literature show that short-term follow-up imaging does not contribute to early detection of false-negative biopsies $(6,11-13,16)$. As for microcalcification-only lesions, since a considerable 


\begin{tabular}{|c|c|c|c|c|}
\hline & Total & 6-months follow-up $(n=194)$ & Without 6-months follow-up $(n=68)$ & Pvalue \\
\hline Age, $y$ & $47.7 \pm 9.0$ & $47.4 \pm 8.7$ & $48.7 \pm 9.8$ & 0.515 \\
\hline Pathologic diagnosis on ST-VAB ${ }^{\text {b }}$ & & & & 0.022 \\
\hline Specific & $20(7.6)$ & $10(5.2)$ & $10(14.7)$ & \\
\hline Non-specific & $242(92.4)$ & $184(94.8)$ & $58(85.3)$ & \\
\hline BI-RADS final assessment category & & & & 0.471 \\
\hline 3 & $3(1.1)$ & $3(1.5)$ & $0(0.0)$ & \\
\hline $4 a$ & $236(90.1)$ & $173(89.2)$ & $63(92.6)$ & \\
\hline $4 \mathrm{~b}$ & $19(7.3)$ & $14(7.2)$ & $5(7.4)$ & \\
\hline $4 \mathrm{C}$ & $4(1.5)$ & $4(2.1)$ & $0(0.0)$ & \\
\hline \multicolumn{5}{|l|}{ Final pathologic diagnosis } \\
\hline Benign & $258(98.5)$ & $191(98.8)$ & $67(99.6)$ & \\
\hline Malignant & $3(1.1)$ & $2(0.8)$ & $1(0.4)$ & \\
\hline High risk lesion & $1(0.4)$ & $1(0.4)$ & $0(0.0)$ & \\
\hline
\end{tabular}

Abbreviations: BI-RADS, breast imaging reporting and data system; SD, standard deviation; ST-VAB, stereotactic vacuum assisted biopsy

${ }^{a}$ Values are expressed as No. (\%) or mean \pm SD.

${ }^{\mathrm{b}}$ Specific: including fibroadenoma, fibroadenomatous hyperplasia, and inflammation; non-specific: including fibrocystic change, adenosis, and columnar cell change

\begin{tabular}{|c|c|c|c|c|c|c|c|c|c|c|}
\hline \multirow[t]{2}{*}{ No. } & \multirow[t]{2}{*}{ Age } & \multicolumn{2}{|c|}{ Imaging features of microcalcifications } & \multirow{2}{*}{$\begin{array}{c}\text { Final } \\
\text { as- } \\
\text { sess- } \\
\text { ment }\end{array}$} & \multirow{2}{*}{$\begin{array}{l}\text { Needle } \\
\text { size, } \\
\text { gauge }\end{array}$} & \multirow{2}{*}{$\begin{array}{l}\text { Initial } \\
\text { pathologic } \\
\text { diagnosis on } \\
\text { biopsy }\end{array}$} & \multirow[t]{2}{*}{ Remnant } & \multirow{2}{*}{$\begin{array}{l}\text { Changes on } \\
\text { mammogra- } \\
\text { phy }\end{array}$} & \multirow{2}{*}{$\begin{array}{l}\text { Follow- } \\
\text { up } \\
\text { interval } \\
\text { to event, } \\
\text { mo }\end{array}$} & \multirow{2}{*}{$\begin{array}{c}\text { Final } \\
\text { patho- } \\
\text { logic } \\
\text { diagno- } \\
\text { sis }\end{array}$} \\
\hline & & Morphology & Distribution & & & & & & & \\
\hline $\mathbf{1}$ & 67 & Amorphous & Grouped & $4 \mathrm{~b}$ & 11 & $\begin{array}{l}\text { Columnar cell } \\
\text { hyperplasia } \\
\text { with microcalci- } \\
\text { fications }\end{array}$ & Yes & $\begin{array}{l}\text { Newly } \\
\text { developed mi- } \\
\text { crocalcifications } \\
\text { near stereotactic } \\
\text { biopsy site }\end{array}$ & 31 & DCIS \\
\hline 2 & 51 & Amorphous & Regional & $4 a$ & 8 & $\begin{array}{l}\text { Papillary ductal } \\
\text { hyperplasia } \\
\text { with microcalci- } \\
\text { fications }\end{array}$ & No & $\begin{array}{l}\text { Newly } \\
\text { developed mi- } \\
\text { crocalcifications } \\
\text { posterior to the } \\
\text { biopsy site }\end{array}$ & 5 & $\begin{array}{c}\mathrm{ADH} \\
(4 \mathrm{~mm})\end{array}$ \\
\hline 3 & 42 & Amorphous & Grouped & $4 \mathrm{a}$ & 8 & $\begin{array}{l}\text { Fibrocystic } \\
\text { change with mi- } \\
\text { crocalcifications }\end{array}$ & Yes & $\begin{array}{l}\text { Newly } \\
\text { developed mi- } \\
\text { crocalcifications } \\
\text { near previous } \\
\text { biopsy site }\end{array}$ & 36 & DCIS \\
\hline 4 & 55 & $\begin{array}{l}\text { Coarse } \\
\text { heterogeneous }\end{array}$ & Grouped & $4 a$ & 8 & $\begin{array}{l}\text { Fibrocystic } \\
\text { change with mi- } \\
\text { crocalcifications }\end{array}$ & Yes & $\begin{array}{l}\text { No change of } \\
\text { the microcalcifi- } \\
\text { cations on } \\
\text { mammography, } \\
\text { newly } \\
\text { developed } \\
\text { suspicious mass } \\
\text { on breast US } \\
\text { performed } 6 \\
\text { months after } \\
\text { biopsy }\end{array}$ & 6 & DCIS \\
\hline
\end{tabular}

Abbreviations: ADH, atypical ductal hyperplasia; DCIS, ductal carcinoma in situ; US, ultrasonography

amount of microcalcifications remain in the breast after stereotactic biopsy, imaging follow-up is critical not to overlook hidden malignancies among the remnant microcalcifications. In our study, the short-term followup mammography did not contribute to detecting the three ductal cancers in situ (DCIS) in our study, among 

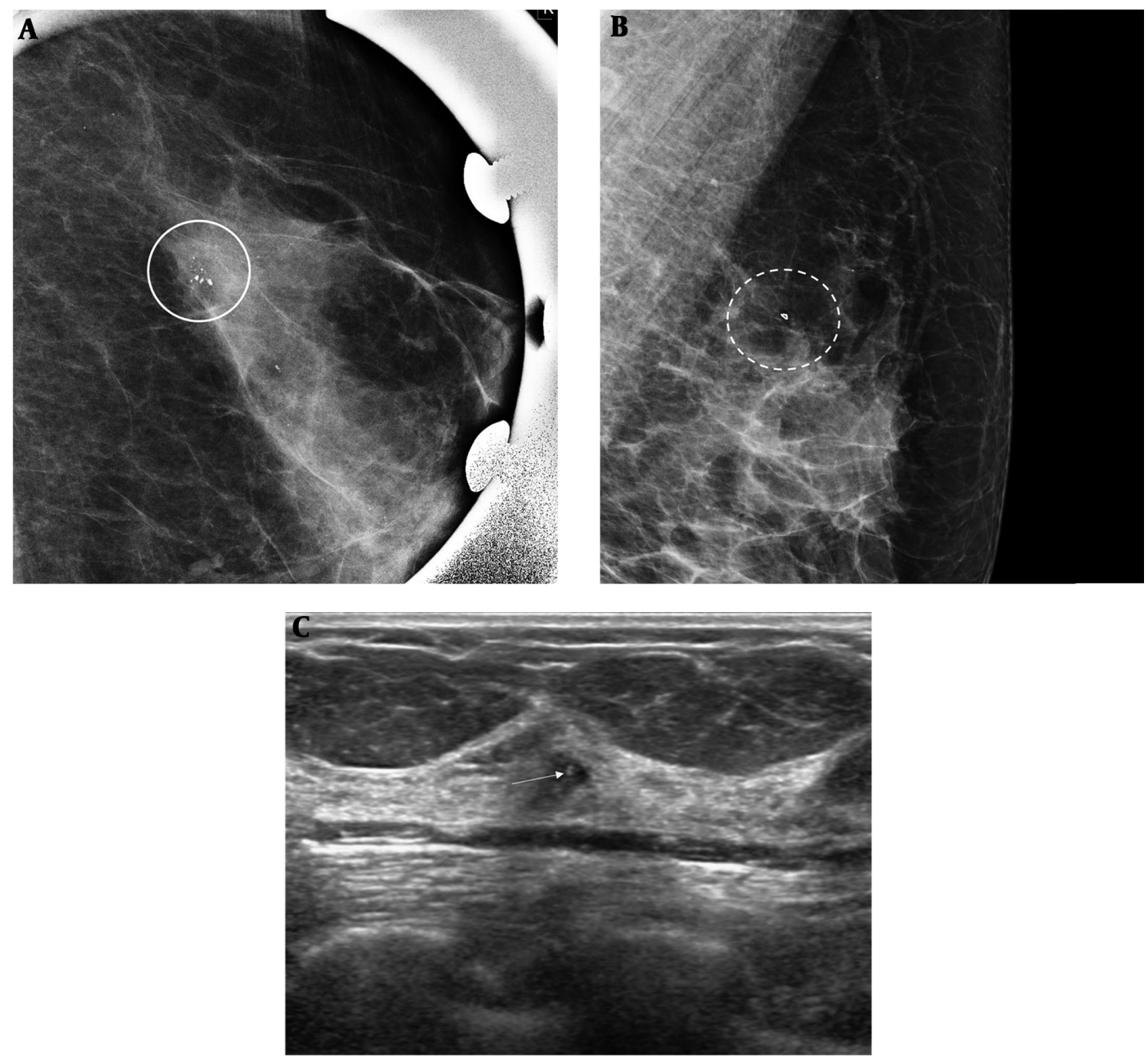

Figure 1. A 63-year-old woman with suspicious microcalcifications in her left breast. A, Magnification mammography of the left breast shows multiple areas of grouped microcalcifications in the left breast, one of which was biopsied (circle), and diagnosed as fibrocystic changes with microcalcifications. B, Follow-up mammography performed 6 months after stereotactic biopsy revealed no remnant microcalcifications at the biopsy site (clip, dotted circle). C, Breast ultrasonography (US) shows a 5-mm sized hypoechoic mass with internal echogenic calcifications (arrow) in the left upper outer quadrant near the biopsy site. This mass was proven as ductal carcinoma in situ (DCIS) on US-guided core biopsy and surgery.

which two of the three additional malignancies manifested as changes on mammography performed 31 and 36 months after biopsy (Table 2). Our results support the other studies in that short-term imaging follow-up after benign stereotactic biopsy results for microcalcifications do not contribute to early detection of cancers, but further studies are anticipated to validate our results.

One of the three additional malignancies detected on imaging follow-up had a newly developed suspicious mass seen on breast US that was performed 6 months after stereotactic biopsy. No changes were seen on the postbiopsy mammography, especially at the prior biopsy site. At our institution, breast US is performed with mammog- raphy for patients scheduled for biopsy and during followup examinations, to rule out the possibility of co-existing US-detectable mass, since US-guided biopsy is easier and more tolerable for the patient. Adding US to patients who are being followed after stereotactic biopsy for benign microcalcifications may contribute to the early detection of false-negative results, but whether US has an added role in detecting additional cancers in patients with biopsyproven benign microcalcifications needs further investigation.

There are several limitations to this study. First, this study was a retrospective study in which selection bias was inevitable. Approximately, $56.7 \%$ of the benign-concordant 

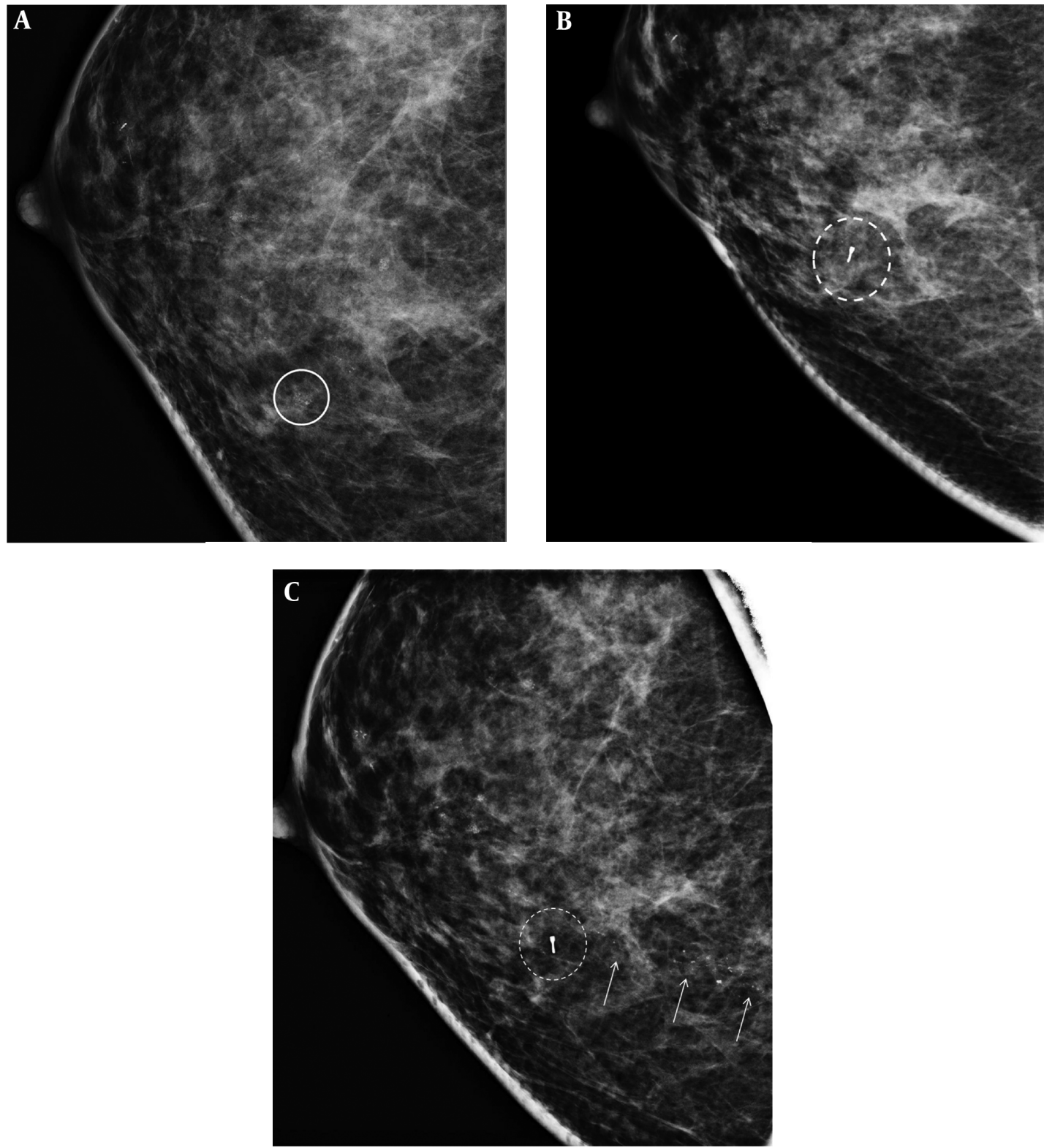

Figure 2. A 50-year-old woman with suspicious microcalcifications in her right breast. A, Magnification mammography of the right breast shows multiple areas of grouped amorphous microcalcifications in the right breast, one of which was biopsied (circle), diagnosed as columnar cell hyperplasia with microcalcifications. B, No significant findings were seen at the biopsy site on the mammogram performed at 6 months after biopsy. C, Follow-up mammography performed 31 months after stereotactic biopsy revealed newly developed fine pleomorphic/fine linear branching microcalcifications (arrows) located posteriorly to the prior biopsy site (dotted circle), which was proven as ductal carcinoma in situ (DCIS) on both stereotactic biopsy and surgery.

microcalcifications were excluded from this study due to the lack of imaging follow-up for more than 2 years. Second, not all patients had undergone short-term imaging follow-up, in which our results may have its limitations in representing the true effectiveness of short-term imaging follow-up. Third, one radiologist retrospectively reviewed the mammography images for microcalcifications. Ob- server variability for microcalcification interpretation was not considered in this study.

In conclusion, the malignancy rate for benign concordant microcalcifications diagnosed with large bore stereotactic biopsy was $1.1 \%$. Short-term follow-up mammography did not contribute in detecting additional breast cancers, and may have a limited role in patients diagnosed 
with benign-concordant microcalcifications on large-bore stereotactic biopsy.

\section{Footnotes}

Authors' Contributions: Guarantor of integrity of the entire study: Eun Kyung Kim and Jung Hyun Yoon; study concepts and design: Jung Hyun Yoon; literature research: Go Eun Yang and Jung Hyun Yoon; clinical studies: Go Eun Yang, Eun Kyung Kim, Min Jung Kim, Hee Jung Moon, Vivian Youngjean Park, and Jung Hyun Yoon; data analysis: Go Eun Yang and Min Jung Kim; statistical analysis: Go Eun Yang and Hee Jung Moon; manuscript preparation: Go Eun Yang and Hyun Yoon; manuscript editing: Eun-Kyung Kim, Min Jung Kim, Hee Jung Moon, Vivian Youngjean Park, and Jung Hyun Yoon

Conflict of Interests: None declared.

Ethical Approval: This study had a retrospective design, and has been approved by the institutional review board of Severance Hospital (IRB No. 4-2017-0502).

Financial Disclosure: None declared.

Funding/Support: This study did not receive any funding from outside agency.

\section{References}

1. Brenner RJ, Fajardo L, Fisher PR, Dershaw DD, Evans WP, Bassett L, et al. Percutaneous core biopsy of the breast: Effect of operator experience and number of samples on diagnostic accuracy. AJR Am J Roentgenol. 1996;166(2):341-6. doi: 10.2214/ajr.166.2.8553943. [PubMed: 8553943].

2. Crystal P, Koretz M, Shcharynsky S, Makarov V, Strano S. Accuracy of sonographically guided 14-gauge core-needle biopsy: Results of 715 consecutive breast biopsies with at least two-year follow-up of benign lesions. J Clin Ultrasound. 2005;33(2):47-52. doi: 10.1002/jcu.20089. [PubMed: 15674836].

3. Schueller G, Jaromi S, Ponhold L, Fuchsjaeger M, Memarsadeghi M, Rudas M, et al. US-guided 14-gauge core-needle breast biopsy: Results of a validation study in 1352 cases. Radiology. 2008;248(2):406-13. doi: 10.1148/radiol.2482071994. [PubMed: 18641246 ].

4. Ohsumi S, Taira N, Takabatake D, Takashima S, Hara F, Takahashi M, et al. Breast biopsy for mammographically detected nonpalpable lesions using a vacuum-assisted biopsy device (Mammotome) and upright-type stereotactic mammography unit without a digital imaging system: Experience of 500 biopsies. Breast Cancer. 2014;21(2):123-7. doi:10.1007/s12282-012-0360-3. [PubMed: 22477266].
5. Bevers TB, Anderson BO, Bonaccio E, Buys S, Daly MB, Dempsey PJ, et al. Breast cancer screening and diagnosis. J Nat Compr Cancer Network. 2009;7(10):1060-96. doi:10.6004/jnccn.2009.0070.

6. Salkowski LR, Fowler AM, Burnside ES, Sisney GA. Utility of 6-month follow-up imaging after a concordant benign breast biopsy result. Radiology. 2011;258(2):380-7. doi: 10.1148/radiol.10091824. [PubMed: 21079199].

7. Youk JH, Kim EK, Kim MJ, Oh KK. Sonographically guided 14-gauge core needle biopsy of breast masses: A review of 2,420 cases with long-term follow-up. AJR Am J Roentgenol. 2008;190(1):202-7. doi: 10.2214/AJR.07.2419. [PubMed: 18094312].

8. Youk JH, Jung I, Kim EK, Kim MJ, Son EJ, Moon HJ, et al. US follow-up protocol in concordant benign result after US-guided 14-gauge core needle breast biopsy. Breast Cancer Res Treat. 2012;132(3):1089-97. doi: 10.1007/s10549-011-1951-y. [PubMed: 22218886].

9. Monticciolo DL, Hajdik RL, Hicks MG, Winford JK, Larkin WR, Vasek JV Jr, et al. Six-month short-interval imaging follow-up for benign concordant core needle biopsy of the breast: Outcomes in 1444 cases with long-term follow-up. AJR Am J Roentgenol. 2016;207(4):912-7. doi: 10.2214/AJR.15.15853. [PubMed: 27340732].

10. Moon HJ, Jung I, Youk JH, Kim MJ, Kim EK. Short-term followup in 6 months is unnecessary for asymptomatic breast lesions with benign concordant results obtained at ultrasonographyguided 14-gauge core needle biopsy. Am J Surg. 2016;211(1):152-8. doi: 10.1016/j.amjsurg.2015.03.036. [PubMed: 26381659].

11. Johnson JM, Johnson AK, O'Meara ES, Miglioretti DL, Geller BM, Hotaling EN, et al. Breast cancer detection with short-interval followup compared with return to annual screening in patients with benign stereotactic or US-guided breast biopsy results. Radiology. 2015;275(1):54-60. doi: 10.1148/radiol.14140036. [PubMed: 25423143]. [PubMed Central: PMC4497520].

12. Manjoros DT, Collett AE, Alberty-Oller JJ, Frazier TG, Barrio AV. The value of 6-month interval imaging after benign radiologicpathologic concordant minimally invasive breast biopsy. Ann Surg Oncol. 2013;20(10):3163-8. doi: 10.1245/s10434-013-3114-3. [PubMed: 23975288].

13. Plecha DM, Garlick C, Dubchuck C, Thompson C, Constantinou $\mathrm{N}$. Comparing cancer detection rates of patients undergoing short term follow-up vs routine follow-up after benign breast biopsies, is follow-up needed? Clin Imaging. 2017;42:37-42. doi: 10.1016/j.clinimag.2016.11.007. [PubMed: 27875760].

14. D'Orsi CJ. ACR BI-RADS Atlas: Breast imaging reporting and data system. American College of Radiology; 2013.

15. Adams MC, Falcon S, Mooney BP, Laronga C, Chau A, Drukteinis JS. Short-term imaging follow-up of patients with concordant benign breast core needle biopsies: Is it really worth it? Diagn Interv Radiol. 2014;20(6):464-9. doi: 10.5152/dir.2014.14023. [PubMed: 25205024]. [PubMed Central: PMC4463290].

16. Jackman RJ, Marzoni FA Jr, Rosenberg J. False-negative diagnoses at stereotactic vacuum-assisted needle breast biopsy: Long-term followup of 1,280 lesions and review of the literature. AJR Am J Roentgenol. 2009;192(2):341-51. doi:10.2214/AJR.08.1127. [PubMed: 19155393]. 